\title{
THE ROLE OF BLOG PSYCHOLOGY IN ONLINE MENTAL HEALTH MOVEMENT: CURRENT STATUS AND IMPLICATIONS
}

\author{
Dennis Relojo \\ Founder, Psychreg, United Kingdom \\ E-mail: dennis.relojo@gmail.com
}

Mental disorders contribute significantly to the global burden of disease, as 4 out of the 10 diseases with the highest burden are psychiatric. About $25 \%$ of all develop one or more psychiatric and behavioural disorders during their lifetime. Unipolar depression ranges as the leading mental disorder with respect to disability adjusted life years. The major psychiatric disorders like schizophrenia and depression are found in all cultures and result in significant disability. As a result, the cost of mental disorders worldwide needs receiving increasing recognition (Kastrup \& Ramos, 2007).

Mental health is a global problem - one that needs greater worldwide attention because until now this has not been achieved. For instance, in the US, the world's sliding superpower, the prisons are the functioning mental-health-care system (Kleinman, 2009). Somewhere between a third and a half of all the homeless people in American cities suffer from mental illness.

Yet balancing this abysmal record, mental healthcare in the US is finally receiving renewed attention and resources aimed at closing the gap in parity with the rest of healthcare. The worst abuses of the mental healthcare system are undergoing near constant, if unsustained, reform and patients' rights are being better, if still not well-protected.

However, treating mental health problem is facing crucial challenges. For one, treatment effectiveness has been shown to vary across subgroups of youth based on characteristics such as comorbidity, problem severity, gender, and age. One study (Bonadio \& Tompsett, 2018) explored if subgroups of youth, identified by applying latent profile analysis to parent-reported symptoms, age, and gender, are better served by specific usual care services. Archival data from a community mental health centre were utilised, including parent-reports of symptoms for 953 youth (44.4\% female; ages $4-18)$ across multiple time points, and services received. Latent profile analysis identified five subgroups including low risk, high risk, internalising, externalising, and delinquent/depressed. The relationship between the intensity level of services and symptoms varied across subgroups. Specifically, high intensity services were related to a greater reduction in symptoms for the internalizing, externalizing, and high risk subgroups.

There is also a crisis among adolescents regarding mental health. Universities UK recently reported a fivefold increase in the number of students disclosing mental health conditions since 2007 (from 9,675 in 2007-2008 to 57, 305 in 2017-2018) and growing pressures on student mental health services, despite only a modest rise in student numbers. A growing number of UK and international studies show that affective disorders in young people are rising substantially, particularly among girls and young women. A recent UK analysis reported a $68 \%$ increase in hospital self-harm presentations in 13-16 year old girls between 2011 (45.9 per 100000) and 2014 (76.9 per 100000). Causes of the escalation are uncertain. Some studies point to a rise in presentation and diagnosis rather than a true increase in incidence. More people self-reporting problems may partly reflect greater willingness to share feelings, such as suicidal thoughts, due to better mental health literacy. If the situation reflects a real deterioration in the mental health of young people, there are several possible explanations (Gunnell, Kidger, \& Elvidge, 2018). 
While we have this problem with mental health, it is interesting to note that more and more Vol. 12, No. 1, 2018 people are drawn to blogs, either as creators or consumers. As Farrell and Drezner (2008) put it: the rise of bloggers raises the vexing question of why blogs have any influence at all, given their relatively low readership and lack of central organisation. We argue that to answer this question we need to focus on two key factors - the unequal distribution of readers across weblogs, and the relatively high readership of blogs among journalists and other political elites. The unequal distribution of readership, combined with internal norms and linking practices allows interesting news and opinions to rise to the 'top' of the blogosphere, and thus to the attention of elite actors, whose understanding of politics may be changed by frames adopted from the blogosphere.

Of particular importance, is the role that blogs play on mental health education. It is remarkable that many teachers today are implementing digital technology in the classroom such as blogging and it is undeniably opening doors to new ways of learning. Teachers who are looking to adopt digital technology should not dismiss the power of the blog to revolutionise learning inside the classroom. Whether it is used for a class website or as stand-alone student projects, blogging in the classroom can easily connect students, parents, and teachers. Encouraging students to blog helps them see connection between what they learn from the class and the different aspects of their lives, and ultimately realise that reflective writing (through blogging) is a worthwhile skill in any field (Relojo, 2018).

Since 1999 blogs have become a significant feature of online culture. They have been heralded as the new guardians of democracy, a revolutionary form of bottom-up news production and a new way of constructing self and doing community in late-modern times. In an article, Hookway (2008) highlights the significance of the blogosphere as a new addition to the qualitative researcher's toolkit and some of the practical, theoretical, and methodological issues that arise from this. Some of the key ethical issues involved in blog data collection are also considered.

As a researcher and mental health advocate, I always have this firm notion that where people spend most their time, that should be used to address an issue. And in this case, most people spend their time consuming blogs, and that could be harnessed to address challenges in global mental health.

As a sub-branch of psychology, blog psychology attempts to apply psychological principles and research in order to optimise the benefits that readers can derive from creating and consuming blogs (Relojo, 2017).

Blogging has come a long way: from being an online craze to becoming a big industry; from being a merely online form of the traditional personal diary to becoming a repository of valuable information. They have become one of the most popular and widely used social platforms of the millennial generation. They enable people around the world to share their stories, connect with each other, and learn more about different topics and themselves. Not only have they given voice to people, but they also play an important role in raising awareness about mental health, helping the most anxious generation of millennials (Williams, 2018).

Blog psychology has an important role to play. Fundamentally, because blogs are vessels of self-reflection. This is demonstrated by Yang (2009). Using the theories of critical reflection and community of practice, the aim of the research was to explore the use of blogs as a reflective platform in the training processes of English as a Foreign Language (EFL) student teachers, who were learning to teach English for future employment in Taiwan. They made use of blogs as a platform to critically reflect on their learning processes as well as to gauge the impact of blogs on their own professional growth. Forty-three student-teachers in two teacher-education programmes at two science and technology institutions in central Taiwan participated in this study.

Two instructors created a blog for use as a discussion forum so that the student-teachers could engage in and examine their own reflection process. The data collected was qualitative in nature, consisting of student teachers' posting messages and comments on the blog, surveys on the student teachers' reflective experiences using blogs as reflection tools, and group reflective dialogues recorded by instructors in class meetings over the implementation of blogs during the course. The results showed that the student-teachers actively discussed teaching theories and their implications through blogs. All of the 43 teachers who took part in the study were reflective, and 
some critically reflected on their thoughts and made significant comments; and the participants considered technology a useful platform for reflecting and communicating with each other.

Recently, there has been a dramatic proliferation in the number of blogs; however, little is published about what motivates people to participate in blog activities (Relojo, 2017). Based on the theory of reasoned action, researchers developed a model involving technology acceptance, knowledge sharing and social influences. A survey of 212 blog participants found strong support for the model. The results indicated that ease of use and enjoyment, and knowledge sharing (altruism and reputation) were positively related to attitude towards blogging and accounted for $78 \%$ of the variance. On the other hand, social factors (community identification) and attitude towards blogging significantly influenced a blog participant's intention to continue to use blogs. Together they explain $83 \%$ of the variance of intention to blog (Hsu \& Lin, 2008).

The phenomenon and practice of blogging offers a rich environment from which to look at the psychology of the internet. By using blogging as a lens, researchers can see that many predictions and findings of early internet research on social and psychological features of computer-mediated communication have held true, whereas others are not as true, and that the psychology of the internet is very much a sense of the one and the many, the individual and the collective, the personal and the political. Blogs illustrate the fusion of key elements of human desire to express one's identity, create community, and structure one's past and present experiences - with the main technological features of 21 st century digital communication. Blogs can serve as a lens to observe the way in which people currently use digital technologies and, in return, transform some of the traditional cultural norms; such as those between the public and the private (Gurak \& Antonijevic, 2008).

With the continued popularity of blogs, it is important that a specialised discipline be developed to encompass all forms of internet-mediated communication, specifically in blogs, such as the use, design, and its impact on mental health and well-being of its readers. Potential theories of blog psychology may include the readers' perception, cognition, and humanistic components in regards to their experience to consuming blogs. Blog psychologists may also draw upon developmental and narrative psychologies and emerging findings from cyberpsychology. The theories and research in psychology could be used as the backbone of blog psychology and guide the discipline itself.

\section{References}

Bonadio, F. T., \& Tompsett, C. (2018). Who benefits from community mental health care? Using latent profile analysis to identify differential treatment outcomes for youth. Journal of Youth and Adolescence, 1-17. https://doi.org/10.1007/s10964-018-0888-4.

Farrell, H., \& Drezner, D. W. (2008). The power and politics of blogs. Public Choice, 134 (1-2), 15-30. https:// doi.org/10.1007/s11127-007-9198-1

Gunnell, D., Kidger, J., \& Elvidge, H. (2018). Adolescent mental health in crisis. BMJ, k2608. https://doi. org/10.1136/bmj.k2608.

Gurak, L. J., \& Antonijevic, S. (2008). The psychology of blogging: You, me, and everyone in between. American Behavioral Scientist, 52 (1), 60-68. https://doi.org/10.1177/0002764208321341.

Hookway, N. (2008). Entering the blogosphere: Some strategies for using blogs in social research. Qualitative Research, 8 (1), 91-113. https://doi.org/10.1177/1468794107085298.

Hsu, C. L., \& Lin, J. C. C. (2008). Acceptance of blog usage: The roles of technology acceptance, social influence and knowledge sharing motivation. Information \& Management, 45 (1), 65-74. https://doi. org/10.1016/j.im.2007.11.001.

Kastrup, M. C., \& Ramos, A. B. (2007). Global mental health. Danish Medical Bulletin, 54 (1), 42-43.

Kleinman, A. (2009). Global mental health: A failure of humanity. The Lancet, 374 (9690), 603-604. https:// doi.org/10.1016/s0140-6736(09)61510-5. 
Relojo, D. (2017). Blog psychology: Insights, benefits, and research agenda on blogs as a dynamic medium to promote the discipline of psychology and allied fields. Psychreg Journal of Psychology, 1 (2), 70-75. https://doi.org/10.5281/zenodo.1289165.

Relojo, D. (2018, February 12). Learning through blogging: Implications of blog psychology in the classroom [Blogpost]. Retrieved from http://psychlearningcurve.org/implications-of-blog-psychology-in-theclassroom/.

Williams, I. (2018, March 16). How conversations on mental health changed for millennials through blogging [Blogpost]. Retrieved from http://chelseakrost.com/how-conversations-on-mental-health-changed-formillennials-through-blogging/sc.

Yang, S. H. (2009). Using blogs to enhance critical reflection and community of practice. Educational Technology \& Society, 12 (2), 11-21.

Received: June 15, 2018

Accepted: June 25, 2018 